\title{
LISTA DE PARECERISTAS AD HOC DE 2017
}

o final de mais um ano, os Editores Associados da revista Educação \& Sociedade e a Diretoria do Centro de Estudos Educaçáo e Sociedade 1 (Cedes) agradecem a colaboraçáo de todos os membros de seus consead hoc elencados a seguir, que emitiram pareceres e promoveram, com sua experiência e profissionalismo, a arbitragem dos textos submetidos para avaliação neste periódico, durante o ano de 2017.

Adalberto Azevedo

Universidade Federal do ABC (UFABC)

Adilson Dalben

Centro Universitário Salesiano de São

Paulo (UNISAL)

\section{Adolfo Ramos Lamar}

Universidade Regional de Blumenau (FURB)

\section{Adriana Bauer}

Fundação Carlos Chagas (FCC)/

Faculdade de Educação da

Universidade de São Paulo (FEUSP)

Adriana Sales de Melo

Universidade de Brasília (UNB)

Adriano de Lemos Alves Peixoto

Universidade Federal da Bahia (UFBA)

Alda Regina Tognini Romaguera

Universidade de Sorocaba (UNISO)

Alessandra Arce

Universidade Federal de São

Carlos (UFSCAR)

Alexandre Simáo Freitas

Universidade Federal de Pernambuco (UFPE)
Ana Elisa Spaolonzi Queiroz Assis

Universidade Estadual de Campinas

(UNICAMP)

\section{Ana Luiza Bustamante Smolka}

Universidade Estadual de Campinas

(UNICAMP)

\section{Ana Maria Fonseca Almeida}

Universidade Estadual de Campinas (UNICAMP)

\section{Ana Paula de Oliveira Corti}

Instituto Federal de Educação, Ciência e Tecnologia de São Paulo (IFSP)

Anderson Ricardo Trevisan

Universidade Estadual de Campinas (UNICAMP)

\section{Andres Eduardo Lainez}

Universidad Pedagógica Nacional

Francisco Morazán (UPNFM)

Anete Abramowicz

Universidade Federal de São

Carlos (UFSCAR)

Ângela Maria Martins

Fundação Carlos Chagas (FCC)

\section{Angelica Cosenza}

Universidade Federal de Juiz de Fora (UFJF)

DOI: 10.1590/ES0101-73302017v38n141LIST 
Antonia Almeida Silva

Universidade Estadual de Feira de Santana (UEFS)

\section{Antônio Agenor Barbosa}

Universidade Federal de Juiz de Fora (UFJF)

Antonio Carlos Rodrigues de Amorin Universidade Estadual de Campinas (UNICAMP)

\section{Ari Fernando Maia}

Universidade Estadual Paulista (UNESP)

Bernardo Mançano Fernandes

Universidade Estadual Paulista (UNESP)

Bernardo Mattes Caprara

universidade Federal da Fronteira Sul (UFFS)

\section{Bianca Thoilliez}

Universidad Autónoma de Madrid (UAM)

\section{Célia Regina Vendramini}

Universidade Federal de Santa

Catarina (UFSC)

\section{Clarice Santos}

Universidade de Brasília (UnB)

Cristiane Machado

Universidade Estadual de Campinas

(UNICAMP)

\section{Daniel Milll}

Universidade Federal de São

Carlos (UFSCAR)

\section{Dante Augusto Galeffi}

Universidade Federal da Bahia (UFBA)

\section{Dante Henrique Moura}

Instituto Federal de Educação, Ciência e Tecnologia do Rio Grande do Norte (IFRN)

\section{Debora Goulart}

Universidade Federal de São Paulo (UNIFESP)

\section{Deise Mancebo}

Universidade do Estado do Rio de Janeiro (UERJ)

\section{Diogo Joel Demarco}

Universidade Federal do Rio Grande do Sul (UFRGS)

\section{Dirce Pacheco Zan}

Universidade Estadual de Campinas

(UNICAMP)

\section{Divino José da Silva}

Universidade Estadual Paulista (UNESP)

Doris Accioly e Silva

Universidade de São Paulo (USP)

Eduardo Pinto e Silva

Universidade Federal de São

Carlos (UFSCAR)

\section{Elaine Prodócimo}

Universidade Estadual de Campinas (UNICAMP)

\section{Eldon Mühl}

Universidade de Passo Fundo (UPF)

\section{Elisabeth Macedo}

Universidade do Estado do Rio de Janeiro (UERJ)

\section{Eliza Bartolozzi Ferreira}

Universidade Federal do Espírito Santo (UFES)

\section{Elizeu Clementino de Souza}

Universidade do Estado da Bahia (UNEB)

\section{Eva da Porta}

Centro de Estudios Avanzados (CEA) Universidad Nacional de Córdoba (UNC)

Fabiana de Cássia Rodrigues

Universidade do Vale do Sapucaí (UNIVAS)

Felipe Hevia

Centro de Investigaciones y Estudios

Superiores en Antropología Social (CIESAS) 
Fernando Luiz Cássio

Universidade Federal do ABC

(UFABC)

\section{Flávia Inês Schilling}

Universidade de São Paulo (USP)

Flavia Pereira Xavier

Universidade Federal de Minas Gerais (UFMG)

\section{Geisa do Socorro C V Mendes}

Pontifícia Universidade Católica de

Campinas (PUC-CAMPINAS)/

Universidade Estadual de Campinas (UNICAMP)

\section{Gilberto Rodrigues}

Universidade Federal do ABC (UFABC)

Gilson Luiz de Oliveira Lima

Centro Universitário Metodista (IPA)

Giselle Cristina Martins Real

Universidade Federal da Grande

Dourados (UFGD)

Guilherme do Val Toledo prado

Universidade Estadual de Campinas (UNICAMP)

\section{Guillermo Ruiz}

Universidad de Buenos Aires (UBA)

\section{Heitor Francisco Heitor de}

Magalhães Souza

Federação de Handebol de Mato

Grasso do Sul (FHMS)

\section{Jefferson Mainardes}

Universidade Estadual de Ponta Grossa (UEPG)

\section{João Cleps Júnior}

Universidade Federal de Uberlândia (UFU)

\section{Joáo Ferreira de Oliveira}

Universidade Federal de Goiás (UFG)
Jorge Alberto Rosa Ribeiro

Universidade Federal do Rio Grande

do Sul (UFRGS)

\section{José Carlos Rothen}

Universidade Federal de São Carlos

(UFSCAR)

\section{Jose Francisco Soares}

Universidade Federal de Minas Gerais (UFMG)

\section{Juan Wahren}

Universdad de Buenos Aires (UBA)

Julio Cabero

Universidade Santiago (US)

Karine Nunes de Moraes

Universidade Federal de Goiás (UFG)

\section{Kimi Tomizaki}

Universidade de São Paulo (USP)

\section{Luis Enrique Aguilar}

Universidade Estadual de Campinas

(UNICAMP)

\section{Leda Aparecida Vanelli Nabuco de}

Gouvea

Universidade Estadual do Oeste do

Paraná (UNIOESTE)

\section{Leda Scheibe}

Universidade Federal de Santa

Catarina (UFSC)/Universidade do

Oeste de Santa Catarina (UNOESC)

\section{Lígia Barros de Freitas}

Universidade Estadual de Minas

Gerais (UEMG)

\section{Luciana Rosa Marques}

Universidade Federal de Pernambuco (UFPE)

\section{Luciano Concheiro}

Universidad Autónoma Metropolitana - Xochimilco (UAM) 
Lucilia Augusta Lino

Universidade do Estado do Rio de Janeiro (UERJ)

\section{Lucília Regina de Souza Machado}

Centro Universitário Privado de Minas (UMA)

\section{Luis Donisete Benzi Grupioni}

Instituto de Pesquisa e Formação

Indígena (IEPE)

\section{Luiz Bezerra Neto}

Universidade Federal de São Carlos (UFSCAR)

\section{Luiz Fernandes Dourado}

Universidade Federal de Goiás (UFG)

\section{Luiz Roberto Gomes}

Universidade Federal de São Carlos (UFSCAR)

\section{Manuel Jacinto Sarmento}

Universidade do Minho (UMINHO)

Márcia Blasi

Faculdade EST (EST)

\section{Maria Carla Corrochano}

Universidade Federal de São

Carlos (UFSCAR)

\section{Marcos Cassin}

Universidade de São Paulo (USP)

Marcos José Silveira Mazzotta

Universidade de São Paulo (USP)

Marcos Garcia Neira

Universidade de São Paulo (USP)

Marcus Vinícius Cunha

Universidade de São Paulo (USP)

Maria Aparecida Bergamaschi

Universidade Federal do Rio Grande do Sul (UFRS)
Maria Clara Di Pierro

Universidade de São Paulo (USP)

Maria Cristina dos Santos Bezerra

Universidade Federal de São Carlos

(UFSCAR)

\section{Maria de Fátima Almeida Martins}

Universidade Federal de Minas Gerais (UFMG)

Maria de Lourdes Zanolli

Universidade Estadual de Campinas

(UNICAMP)

\section{Maria Isabel Antunes Rocha}

Universidade Federal de Minas Gerais (UFMG)

\section{Maria Margarida Machado}

Universidade Federal de Goiás (UFG)

Maria Ribeiro do Valle

Universidade Estadual Paulista (UNESP)

\section{Maria Rita Neto Sales Oliveira}

Centro Federal de Educação Tecnológica de Minas Gerais (CEFET-MG)

\section{Maria-Beatriz Moreira Luce}

Universidade Federal do Rio Grande do Sul (UFRGS)

Mario Luiz Neves de Azevedo

Universidade Estadual de Maringá (UEM)

\section{Mônica Castagna Molina}

Universidade de Brasília (UNB)

\section{Monica Fantin}

Universidade Federal de Santa

Catarina (UFSC)

\section{Monica Ribeiro da Silva}

Universidade Federal do Paraná (UFPR)

Myriam Inés Feldfeber

Universidad de Buenos Aires (UBA) 
Nathalia Cassetari

Universidade de Brasília (UNB)

Nelson Cardoso do Amaral

Universidade Federal de Goiás (UFG)

Nilson Marcos Dias Garcia

Universidade Tecnológica Federal do

Paraná (UTFPR)

Otaviano Augusto Marcondes Helene

Universidade de São Paulo (USP)

Pablo Álvarez Domíguez

Universidade Santiago (US)

Paulo Henrique Arcas

Instituto Internacional de Planeamiento de la Educación (IIPE UNESCO)

Paulo Henrique Fernandes

Universidade Federal de Pernambuco (UFPE)

\section{Pedro Angelo Pagni}

Universidade Estadual Paulista (UNESP)

\section{Rafael de Brito Dias}

Universidade Estadual de Campinas

(UNICAMP)

\section{Raimundo Luiz Silva Araujo}

Universidade de Brasília (UnB)

Ramon de Oliveria

Universidade Federal de Pernambuco (UFPE)

\section{Regiane Helena Bertagna}

Universidade Estadual Paulista Júlio de Mesquita Filho (UNESP)

\section{Régis Henrique dos Reis Silva}

Universidade Estadual de Campinas (UNICAMP)

Robert Evan Verhine

Universidade Federal da Bahia (UFBA)
Sandro Vinicius Sales dos Santos

Universidade Federal dos Vales do

Jequitinhonha e Mucuri (UFVJM)

\section{Sergio Stoco}

Universidade Estadual de Campinas

(UNICAMP)

Sônia Aparecida Siquelli

Universidade do Vale do Sapucaí

(UNIVAS)

Sônia Maria Portella Kruppa

Universidade de São Paulo (USP)

Teise Oliveira Guaranha Garcia

Universidade de São Paulo (USP)

Vanessa Regina de Oliveira Martins

Universidade Federal de São Carlos

(UFSCAR)

Vânia Gomes Zuin

Universidade Federal de São Carlos

(UFSCAR)

Vera Lúcia Jacob Chaves

Universidade Federal do Pará (UFPA)

Waldemar Marques

Universidade de Sorocaba (UNISO)

Willian Simóes

Universidade Federal da Fronteira Sul (UFFS)

\section{Vilma Nista}

Universidade de Sorocaba (UNISO)

\section{Wilson Alviano Jr}

Universidade Federal de Juiz de Fora (UFJF)

Wilson Mesquita de Almeida

Universidade Federal do ABC

(UFABC)

Wivian Weller

Universidade de Brasília (UnB) 